\title{
Should MASP-2 Deficiency Be Considered a Primary Immunodeficiency? Relevance of the Lectin Pathway
}

\author{
M. Isabel García-Laorden 1,2 • Elisa Hernández-Brito ${ }^{3,4}$ - Carmen Muñoz-Almagro 5,6,7 • \\ Svetlana Pavlovic-Nesic ${ }^{8}$ • Iñigo Rúa-Figueroa ${ }^{9}$ - M. Luisa Briones ${ }^{10}$ • Olga Rajas ${ }^{11}$. \\ Luis Borderías ${ }^{12} \cdot$ Antoni Payeras $^{13} \cdot$ Leonardo Lorente $^{14}$. Jordi Freixinet ${ }^{15}$. Jose Ferreres ${ }^{16}$. \\ Ignacio Obando ${ }^{17}$. Nereida González-Quevedo ${ }^{3}$. Felipe Rodríguez de Castro ${ }^{4,18}$. \\ Jordi Solé-Violán ${ }^{1,19} \cdot$ Carlos Rodríguez-Gallego ${ }^{3,20}$
}

Received: 15 June 2019 / Accepted: 29 October 2019 / Published online: 11 December 2019

(C) Springer Science+Business Media, LLC, part of Springer Nature 2019

\begin{abstract}
Mannose-binding lectin (MBL)-associated serine protease-2 (MASP-2) is an indispensable enzyme for the activation of the lectin pathway of complement. Its deficiency is classified as a primary immunodeficiency associated to pyogenic bacterial infections, inflammatory lung disease, and autoimmunity. In Europeans, MASP-2 deficiency, due to homozygosity for $c .359 \mathrm{~A}>G$ (p.D120G), occurs in 7 to $14 / 10,000$ individuals. We analyzed the presence of the p.D120G mutation in adults (increasing the sample size of our previous studies) and children. Different groups of patients (1495 adults hospitalized with communityacquired pneumonia, 186 adults with systemic lupus erythematosus, 103 pediatric patients with invasive pneumococcal disease) and control individuals (1119 healthy adult volunteers, 520 adult patients without history of relevant infectious diseases, and a pediatric control group of 311 individuals) were studied. Besides our previously reported MASP-2-deficient healthy adults, we found a new p.D120G homozygous individual from the pediatric control group. We also reviewed p.D120G homozygous individuals reported so far: a total of eleven patients with a highly heterogeneous range of disorders and nine healthy controls (including our four MASP-2-deficient individuals) have been identified by chance in association studies. Individuals with complete deficiencies of several pattern recognition molecules of the lectin pathway (MBL, collectin-10 and collectin-11, and ficolin-3) as well as of MASP-1 and MASP-3 have also been reviewed. Cumulative evidence suggests that MASP-2, and even other components of the LP, are largely redundant in human defenses and that individuals with MASP-2 deficiency do not seem to be particularly prone to infectious or autoimmune diseases.
\end{abstract}

Keywords MASP- $2 \cdot \mathrm{MBL} \cdot$ primary immunodeficiency $\cdot$ complement $\cdot$ lectin pathway $\cdot$ ficolin- $3 \cdot$ collectin

\section{Introduction}

Deficiencies in components of the classical and alternative pathways of the complement system are associated to bacterial infections, particularly by Streptococcus pneumoniae, Haemophilus influenzae, and Neisseria spp., particularly N. meningitidis, as well as to autoimmune, mainly rheumatic, diseases $[1,2]$. The role of deficiencies of the components of the lectin pathway (LP)

M. Isabel García-Laorden and Elisa Hernández-Brito contributed equally to this work

Carlos Rodríguez-Gallego

jrodgal@gobiernodecanarias.org

Extended author information available on the last page of the article on predisposition to infection and autoimmunity is controversial [3]. The LP is triggered by the pattern recognition molecules (PRMs), mannose-binding lectin (MBL); ficolin-1, ficolin-2, and ficolin-3; and collectin (CL)-10 and CL-11 after binding to surfaces displaying various arrays of carbohydrates or acetyl groups [3]. These PRMs circulate in heterocomplexes with MBL-associated serine proteases (MASPs), which initiate the proteolytic cascade of the LP after binding of the PRM to their ligands. There are three MASPs, MASP-1, MASP-2, and MASP-3. MASP-2 is, together with MASP-1, indispensable for the activation of the LP (Fig. 1) [3-5]. Both activated MASP-1 and MASP-2 can cleave C2, but C4 is cleaved only by MASP-2. In the absence of MASP-1 or MASP-2, the classical $\mathrm{C} 3$ convertase $\mathrm{C} 4 \mathrm{bC} 2 \mathrm{a}$ cannot be generated through the $\mathrm{LP}$, and no LP activation is observed. 


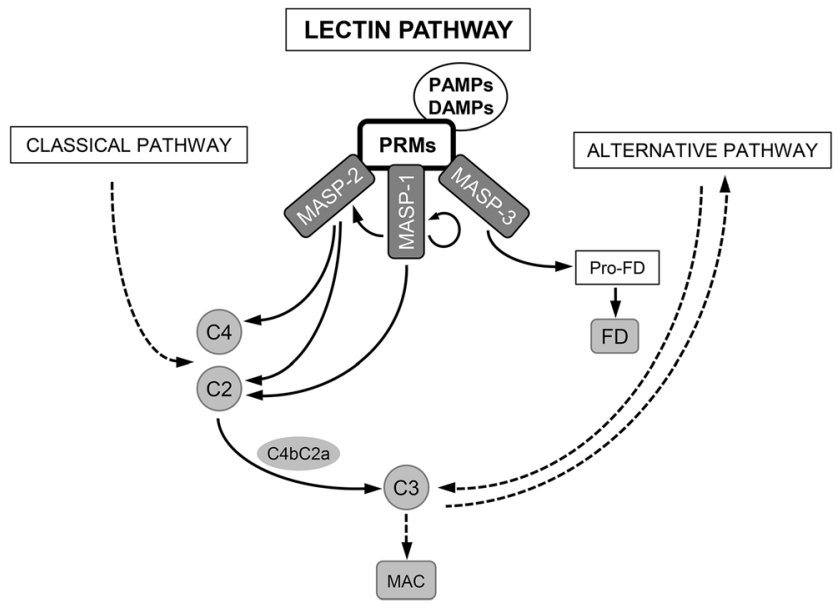

Pattern recognition molecules (PRM) of the Lectin Pathway:

MBL, FCN-1, FCN-2, FCN-3 and CL-LK (heterocomplex of CL-10 and CL-11)

Fig. 1 The lectin pathway (LP) initiates after binding of pattern recognition molecules (PRMs) to surfaces displaying various arrays of carbohydrates or acetyl groups in PAMPs (pathogen-associated molecular patterns) or DAMPs (damage-associated molecular patterns). Several PRM of the LP have been identified so far: mannose-binding lectin; ficolin-1, ficolin-2, and ficolin-3; and collectin (CL)-10 and CL11. CL-10 and CL-11 were found to circulate as heteromeric complexes (CL-LK) of one CL-L1 and two CL-K1 polypeptide chains, able to activate the LP. The serine proteases that initiate the proteolytic cascade of the LP are the MBL-associated serine proteases (MASPs), which circulate in complex with the PRM. When the PRMs of the LP bind to the target surface, zymogen MASP-1 autoactivates first and then activates zymogen MASP-2. Both activated MASP-1 and MASP-2 can cleave C2, but $\mathrm{C} 4$ is cleaved only by MASP-2. In the absence of MASP-1 or MASP2 , the classical $\mathrm{C} 3$ convertase $\mathrm{C} 4 \mathrm{bC} 2 \mathrm{a}$ cannot be generated through the LP, and no LP activation is observed. MASP-3 is the primary physiological activator of pro-factor D under resting conditions in human blood, inducing the activation of the alternative pathway, which serves as an amplification loop for the classical and the lectin pathways. Activation of MASP- 3 by MASP-1 and MASP- 2 has been also proposed. In addition, it was recently shown that MASP- 2 can directly cleave $\mathrm{C} 3$ in the absence of $\mathrm{C} 4$ and/or $\mathrm{C} 2$ on LP-activating surfaces [3-5]. PAMPS pathogen-associated molecular patterns, DAMPS damage-associated molecular patterns, $P R M$ pattern recognition molecules, MASP MBLassociated serine proteases, $M B L$ mannose-binding lectin, $F C N-1$ ficolin-1 (also commonly termed M-ficolin), $F C N$-2 ficolin-2 (initially identified as L-ficolin), $F C N-3$ ficolin-3 (initially identified as $\mathrm{H}$ ficolin), $C L-10$ collectin-10 (also known as collectin liver 1, CL-L1), $C L-11$ collectin-11 (also known as collectin kidney 1, CL-K1), FD factor D, MAC membrane attack complex

Deficiency of MASP-2 was first reported in 2003 [6] in an adult individual suffering from autoimmune and inflammatory disorders, as well as severe and recurrent pneumococcal infections. This patient, who was totally deficient in LP activity, was found to be homozygous for a mutation in exon 3 of the MASP2 gene (rs72550870, p.D120G, c.359A > G). This mutation results in a significant decrease in serum levels of the protein, reaching very low levels in homozygous individuals [6,7]. But, more importantly, it abrogates the LP activation by preventing the interaction of MASP-2 with the PRMs of the LP [6].
Since 2004, MASP-2 deficiency has been included in the classification of primary immunodeficiencies (PID) as a complement deficiency associated to pyogenic infections, inflammatory lung disease, and autoimmunity [2, 8]. In 2006 and 2008, we reported three MASP-2-deficient healthy adults homozygous for p.D120G in MASP2 [7, 9], casting doubts about the clinical penetrance of this deficiency. Other deficient individuals, both healthy subjects and patients with a heterogeneous variety of disorders, have also been reported.

In order to shed light on the clinical consequences of MASP-2 deficiency, we have analyzed the presence of the p.D120G mutation in different groups of adult and pediatric patients and healthy individuals. In addition, we have reviewed MASP2 p.D120G homozygous individuals reported so far.

\section{Methods}

\section{Patients and Controls}

The present study analyzes different groups of patients and healthy individuals. We have increased the sample size of the adult groups published in our previous studies [7, 9]. In addition, we have included pediatric patients with invasive pneumococcal disease (IPD), as well as a pediatric control group. The groups of adult individuals included in the present study comprise 1495 patients hospitalized with communityacquired pneumonia (CAP, 461 with pneumococcal CAP) from 6 Spanish hospitals (mean age, $62.8 \pm 17.7$ years, $33.9 \%$ females), a control group of 520 patients without history of relevant infectious diseases from the same origin than CAP patients (mean age $61.5 \pm 13.9$ years, $34.4 \%$ females), 1119 unrelated healthy volunteers (blood and bone marrow donors and hospital staff) (mean age $40.9 \pm 14.7$ years, $51.8 \%$ females), and 186 patients with systemic lupus erythematosus (SLE) (mean age $45.8 \pm 12.3$ years, $91.9 \%$ females). The pediatric groups analyzed are composed of 103 patients with IPD (mean age $2.9 \pm 2.5$ years, $36.5 \%$ females) from 3 Spanish hospitals, as well as 311 children from a control group (patients attended for minor surgery with no history of respiratory diseases and without occurrence of inflammatory processes in at least 3 months previous to their inclusion) (mean age $12.7 \pm 13.2$ months, $67.2 \%$ females). Individuals with ancestors other than Spanish were excluded from the study.

The diagnosis of CAP was based on the presence of acute onset of signs and symptoms suggestive of lower respiratory tract infection and on radiographic evidence of a new pulmonary infiltrate with no alternative known cause. IPD was considered in cases of infection requiring hospitalization and confirmed when S. pneumoniae was detected by culture or molecular methods from a normally sterile site (blood, cerebrospinal, synovial or pleural fluid, but not sputum). Patients with 
SLE were diagnosed according to the American College of Rheumatology 1997 modified criteria [10].

Analysis was done as part of research projects originally approved by the Ethic Committee of the Hospital Universitario de Gran Canaria Doctor Negrin, Canary Islands Spain, and Hospital Sant Joan de Deu, Barcelona, Spain, and informed consent was obtained from all individuals (or representative). Research reported in the manuscript is in compliance with the Helsinki Declaration.

\section{Genotyping}

Genomic DNA was isolated from whole blood according to standard phenol-chloroform procedure or by the means of automated nucleic acid purification systems (MagNA Pure, Roche Molecular Diagnostics, Pleasanton, CA, USA, or iPrep, Invitrogen by Life Technologies, Carlsbad, CA, USA, or Nuclisens EasyMag System, Biomerieux, Marcy l'Etoile, France). MASP2 p.D120G mutation was analyzed by PCR-RFLP as previously described [9]. Homozygous individuals were confirmed by Sanger sequencing with a different set of primers $[7,9]$.

\section{Identification of Previous Cases}

MASP2 p.D120G homozygous individuals previously reported were identified by extensive searching in PubMed, using the search terms "mannose-binding lectin-associated serine protease 2," "mannose-binding protein-associated serine protease 2," "mannan-binding lectin-associated serine protease 2," "MASP-2," and "MASP2."

\section{Statistical Analysis}

Frequencies of the different genotypes are presented as number of individuals and percentage from total. Expected numbers of
MASP2 p.D120G homozygous individuals were calculated based on the frequency of the mutated allele. Comparison of $M A S P 2$ genotypes was performed using the Chi-square test or Fisher's exact test when needed, considering statistical significance when $\mathrm{P}<0.05$. Statistical analysis was performed using SPSS 15.0 (SPSS Inc., Chicago, IL, USA).

\section{Results}

Table 1 shows the frequency of the genotypes of the $c .359 A>$ $G$ variant (p.D120G) of the MASP2 gene in the studied populations. We had previously reported three MASP-2-deficient individuals with no history of serious infections or autoimmunity [7, 9]. A new MASP2 p.D120G homozygous individual has now been found in the control group of children without records of respiratory diseases. Six episodes of otitis were documented during his first 2 years of life (three of the episodes were attended at primary care centers, and only one of them was suppurative). Between the age of 3 and 5, he had two episodes of otitis per year; only the first of them was treated with antibiotics. This individual is currently 12 years old, and no further episodes of otitis or other diseases have been recorded. He has never fulfilled diagnostic criteria of PID.

We have not found any MASP2 p.D120G homozygote among 1495 adults with CAP (of which 73 had pleural empyema, 470 severe sepsis, 79 acute respiratory distress syndrome, and 461 were diagnosed with pneumococcal CAP), 103 children with IPD, and 186 patients with SLE (30 of them with at least one episode of pneumonia). No differences in genotypic or allelic frequencies were found when comparing the groups of patients to their control populations. The expected frequency of homozygosity for the p.D120G variant of our two healthy control populations together (adults and children, $\mathrm{N}=1430$ ) is of 6.9 in every 10,000 individuals.
Table 1 Genotypes of the $M A S P 2$ p.D120G mutation $(A>$ $G)$ in the groups of individuals analyzed

\begin{tabular}{llllll}
\hline & $N$ & $A / A$ & $A / G$ & $G / G$ & Expected $G / G^{1}$ \\
\hline Healthy adults & 1119 & $1063(95.00)$ & $53(4.74)$ & $3(0.27)$ & $0.78(0.07)$ \\
Adult no infection controls $^{2}$ & 520 & $484(93.08)$ & $36(6.92)$ & $0(0)$ & $0.62(0.12)$ \\
Children no infection controls $^{3}$ & 311 & $296(95.18)$ & $14(4.50)$ & $1(0.32)$ & $0.21(0.07)$ \\
Adult CAP patients & 1495 & $1414(94.58)$ & $81(5.42)$ & $0(0)$ & $1.10(0.07)$ \\
Children with IPD & 103 & $100(97.09)$ & $3(2.91)$ & $0(0)$ & $0.02(0.02)$ \\
SLE patients & 186 & $174(93.55)$ & $12(6.45)$ & $0(0)$ & $0.19(0.1)$ \\
\hline
\end{tabular}

Values are number of individuals (\%). CAP community-acquired pneumonia (including 73 patients with pleural empyema, 470 with severe sepsis, and 79 with acute respiratory distress syndrome), IPD invasive pneumococcal disease, SLE systemic lupus erythematosus

${ }^{1}$ Number of expected individuals homozygous for the $\mathrm{G}$ allele (\%)

${ }^{2}$ Control group of 520 patients without history of relevant infectious diseases from the same origin as CAP patients

${ }^{3}$ Control group of children attended for minor surgery with no history of relevant respiratory diseases and without occurrence of inflammatory processes in at least 3 months previous to their inclusion 
Table 2 shows the eleven p.D120G homozygous patients reported in the scientific literature so far. Besides the first patient reported in 2003 [6], ten additional p.D120G homozygous patients (three children and seven adults) have been subsequently identified by chance while performing association studies. These patients presented with a heterogeneous range of diseases: two patients suffered from recurrent respiratory infections [11-13], another one suffered from cystic fibrosis [14], two others were admitted to a lung clinic (no specific disease was reported) [15], two more suffered from pulmonary tuberculosis [13], one patient had colorectal cancer [16], another one was an hepatitis $\mathrm{C}$ virus positive individual with hepatocellular carcinoma [17], and the last one was a previously healthy female, aged 66 and with no particular trend to infection, who was hospitalized at general ward due to nonsevere CAP [18]. Noteworthy, nine healthy individuals (two children and seven adults) homozygous for p.D120G, including our four cases, have also been found in genetic association studies (Table 3) [7, 9, 13, 17, 20, 21].

\section{Discussion}

We have performed an extensive study aimed to analyze MASP-2 deficiency due to the p.D120G variant in a large group of patients with CAP, IPD, or SLE and a large group of controls (both children and adults). Besides our three previously reported healthy individuals with MASP-2 deficiency [7, 9], we herein report another MASP2 p.D120G homozygous individual who does not present particularly severe or recurrent infections, nor inflammatory or autoimmune diseases, or any other relevant disorder.

The study of individuals with PID has proven to be an important source of information on the relevance of the affected molecules in humans in nature [23]. The first case of MASP-2 deficiency, described in 2003 by Stengaard-Pedersen et al [6], suggested that this was a new monogenic defect underlying an autosomal recessive immunodeficiency, predisposing to severe infections and autoimmunity. It is worth noting that this individual also showed low levels of $\mathrm{Clq}$ (with presence of anti-Clq autoantibodies), C3, and C4 and severely impaired classical and alternative pathways activity. This patient was identified when sera from 125 patients with suspected PID were analyzed for MBL-dependent LP activity deficiency [6, 22]. Ten additional p.D120G homozygous patients (three children and seven adults), with a heterogeneous range of diseases, have been identified by chance in subsequent association studies [11-18] (Table 3). However, nine healthy individuals (two children and seven adults) homozygous for p.D120G have also been found by chance in genetic association studies [7, 9, 13, 17, 19-21, and this study]. Taken together, these findings suggest that MASP-2 deficiency no longer fulfills the premise that to establish a causal relationship between a genotype and a clinical phenotype, the genotype must not occur in healthy individuals [24]. Certainly, some PID show an incomplete penetrance, and healthy individuals with causative genotypes have been identified in familial studies. In any event, incomplete penetrance through life is the exception rather than the rule for most PID, particularly for those PID with autosomal recessive inheritance [23].

Since 2004, MASP-2 deficiency has been included in the classification of PID as a complement deficiency associated to high susceptibility to recurrent pyogenic infections, as well as to inflammatory lung disease and autoimmunity $[2,8,25]$. C1q, $\mathrm{C} 2$, and $\mathrm{C} 4$ deficiencies are also included as PID but, in this case, with low susceptibility to pyogenic infection and to SLE. This seems paradoxical, since approximately $50 \%$ of $\mathrm{Clq}$-deficient individuals present with significant infections by encapsulated bacteria, around $50 \%$ of those with $\mathrm{C} 2$ deficiency have invasive infections, particularly by pneumococcus, and $76 \%$ of $\mathrm{C} 4$ deficient patients suffer from recurrent respiratory infections, including pneumonia and meningitis [1, 26-28]. Likewise, 80-95\%, $25 \%$, and $76 \%$ of individuals with deficiency of $\mathrm{C} 1 \mathrm{q}, \mathrm{C} 2$, and
Table 2 Reported patients with MASP-2 deficiency due to homozygosity for the p.D120G mutation

\begin{tabular}{lll}
\hline Reported MASP2 p.D120G homozygous individuals & Year & Ref \\
\hline $\begin{array}{l}\text { Patients (source) } \\
\text { 1. Adult Danish individual with several autoimmune manifestations, ulcerative colitis, and } \\
\quad \text { frequent infections, including severe pneumococcal pneumonias }\end{array}$ & 2003 & {$[6,22]$} \\
2. Pediatric Polish individual with recurrent pneumonias (from a study of 335 Polish children & 2004 & {$[11,13]$} \\
$\quad$ with RRI) & 2006 & {$[14]$} \\
3. Pediatric Danish individual with CF (from a group of 109 patients with CF) & 2007 & {$[15]$} \\
4. Adult Danish individual admitted to the Lung Clinics (n.a.) & 2007 & {$[15]$} \\
5. Adult Danish individual admitted to the Lung Clinics (n.a.) & 2008 & {$[17]$} \\
6. HCV ${ }^{+}$Italian adult individual with HC (from a study of 215 adult patients with HC) & 2009 & {$[13]$} \\
7. Pediatric Polish individual with RRI and skin abscesses (from a study of 331 children with & & \\
$\quad$ allergy and/or RRI) & 2011 & {$[16]$} \\
8. Adult Danish individual with colorectal cancer (from a study of 593 patients with colorectal cancer) \\
9. Adult Polish individual with pulmonary TB (from a study of 440 TB patients) & 2015 & {$[13]$} \\
10. Adult Polish individual with pulmonary TB (from a study of 440 TB patients) & 2015 & {$[13]$} \\
11. Adult Norwegian individual with non-severe CAP (from a study of 257 adult CAP patients) & 2018 & {$[18]$} \\
\hline
\end{tabular}

$R R I$ recurrent respiratory infection, $C F$ cystic fibrosis, $H C$ hepatocellular carcinoma, $H C V$ hepatitis $\mathrm{C}$ virus, $T B$ tuberculosis, CAP community-acquired pneumonia, n.a. not available 
Table 3 Reported healthy individuals with MASP-2 deficiency due to homozygosity for the p.D120G mutation

\begin{tabular}{lll}
\hline Reported MASP2 p.D120G homozygous individuals & Year & Ref \\
\hline $\begin{array}{l}\text { 1. Adult British individual (from a study of } 314 \text { family-based trios with offspring affected with psoriasis) } \\
\text { 2. Adult Spanish individual (from a control group of } 868 \text { healthy adults in a study of adult CAP patients, }\end{array}$ & 2005 & {$[19]$} \\
adult SLE patients, and children with RRI) & {$[9]$} \\
$\begin{array}{l}\text { 3. Adult Spanish individual (from a control group of } 868 \text { healthy adults in a study of adult CAP patients, } \\
\text { adult SLE patients, and children with RRI) }\end{array}$ & 2006 & {$[9]$} \\
4. Adult Spanish individual (from a control group of 805 healthy adults in a study of adult CAP patients & 2008 & {$[7]$} \\
and patients without relevant infectious diseases) & 2008 & {$[17]$} \\
5. Adult Italian individual (from a control group of 162 healthy individuals for a study of adult patients \\
with HC) & 2014 & {$[20]$} \\
6. Adult Polish individual (from a study of 179 children with dental caries) & 2015 & {$[13]$} \\
7. Adult Polish individual (from a control group of 276 healthy individuals for a study of TB patients) \\
8. Pre-term Polish newborn (from a control group of 273 healthy newborns for a study of children with \\
$\quad$ sepsis and non-septic infected babies) & 2016 & {$[21]$} \\
9. Pediatric Spanish individual (from a control group of children with no history of respiratory diseases) & (current study) \\
\hline
\end{tabular}

$C A P$ community-acquired pneumonia, $S L E$ systemic lupus erythematosus, $R R I$ recurrent respiratory infection, $H C$ hepatocellular carcinoma, $T B$ tuberculosis
C4, respectively, are diagnosed with SLE, and another $18 \%$ of C2-deficient patients suffer from undifferentiated connective tissue disease or vasculitis [1, 26-28].

Several deficiencies of other components of the LP may shed light on the role of MASP-2 deficiency and LP deficiencies in general, in the susceptibility to infectious and autoimmune diseases. MBL deficiency was originally included in the classification of PID as a complement deficiency associated to pyogenic infections, although its very low clinical penetrance was stated [8]. More than $5 \%$ of individuals lack functional serum MBL, mostly due to homozygosity or compound heterozygosity for three common mutations in exon 1 of the MBL2 gene [3]. It was even proposed that the high worldwide frequencies of MBL-deficient alleles may be due to random drift, underscoring the evolutionary neutrality of MBL2 [29]. These data also suggested that MBL is largely redundant in human defenses [23, 29]. As further large studies were carried out, controversial data about the role of MBL deficiency in predisposition to severe infection, particularly to severe sepsis and to meningococcal and pneumococcal infection, were reported [3, 7, 23, 30-37]. Overall, although a role as a modifying factor cannot be excluded, the results suggest that the deficiency is mostly asymptomatic and, in 2011's update of PID [38], it was no longer included. Human and mouse CL-11, but not MBL, drive LP activation on the surface of $S$. pneumoniae and other bacteria, and it increases opsonophagocytosis [39-42]. Patients with autosomal recessive CL-11 or CL-10 deficiency suffer from Malpuech-MichelsMingarelli-Carnevale (3MC) syndrome, characterized by craniofacial, genital, and often mental defects. However, CL-11- and CL-10-deficient patients, who lack CL-11 or CL-10 secretion, do not manifest a predisposition to infection, autoimmunity, or another immunological phenotype [41, 43-46]. Mutations at the MASP1 gene were also identified in patients with 3MC syndrome [41, 43, 45, 47, 48]. The MASP1 gene gives rise to three alternative splice products, MASP-1, MASP-3, and Map-44, which share the first eight exons. However the serine protease domains of MASP-1 (exons 13-18) and MASP-3 (exon 12) are coded by different exons (no serine protease domain is present in Map-44) [3-5]. Most mutations in MASP1 in patients with 3MC syndrome lie in the exon 12, unique to MASP-3. However, in nine patients, the mutations disrupt the three protein products [45-48]. Nevertheless, no infectious or autoimmune phenotype was documented in these patients despite the undetectable LP activity due to the absence of MASP-1 [48, 49]. The first patient with ficolin-3 deficiency was reported in 2009 when ficolin-3 levels were measured in a large series of patients with suspected immunodeficiencies [50]. The patient was an adult with recurrent respiratory tract infections and cerebral abscesses. However, he had underwent splenectomy at the age of 20 due to thrombocytopenia, and he had partial IgM deficiency and a low response to pneumococcal polysaccharide vaccine. The deficiency was due to a mutation that is estimated to occur in homozygosity in 1/10,000 individuals. Ficolin-3 deficiency was also considered a PID associated to high susceptibility to recurrent pyogenic infections and to abscesses [2, 25]. Five additional patients with a rather heterogeneous range of clinical manifestations and a healthy control were later found by chance in association studies to have genetically proven ficolin-3 deficiency $[51,52]$; a role as a predisposing factor for SLE was recently suggested [52]. Nevertheless, the first reported patient [50] was later diagnosed with genetically proven Wiskott-Aldrich syndrome [3].

MASP-2 deficiency due to homozygosity for p.D120G is estimated to occur approximately in 7 (as in the current study) to 15 in every 10,000 individuals of European origin [14, 21]. This frequency would make MASP-2 deficiency the second most common PID after IgA deficiency, provided that MBL deficiency is not a PID. Nevertheless, MASP-2 deficiency, as the MBL deficiency, seems to merely be a consequence of a common polymorphism,

Our study has several limitations. In our group of CAP patients, most individuals suffered from one only episode of CAP, and hence it does not represent a PID cohort. In addition, patients with a diagnosed PID were excluded from our study, and genetic or immunological studies aimed to the diagnosis of PID were not 
carried out to most, particularly adult, patients and controls. A role of MASP-2 deficiency as a potential disease modifier in individuals with additional inherited immune deficiencies or in patients with underlying diseases impairing immune defense mechanisms cannot be ruled out [3]. We cannot either rule out that some of the MASP-2-deficient individuals, even healthy controls, had not yet manifested the disease phenotype. In any event, health status was evaluated in at least four healthy controls aged 19 [20], 29 [13], 37 [9], and 39 [9] years. Remarkably, the 37-year-old healthy control female, a member of the hospital staff [9], is currently 51 years old, and she remains healthy. Conversely, an excess of LP activation might be also harmful due to the possibility of an unbalanced proinflammatory response and an additional host injury. Indeed, the LP, particularly MASP2, may behave as a double-edged sword and be even detrimental in serious disease conditions such as myocardial infarction, stroke, renal ischemia-reperfusion injury, and rheumatoid arthritis $[4,5,41,53]$. Interestingly, increased levels of MASP-2 may contribute to poor disease outcome of pneumococcal meningitis [54]. Due to the low frequency of the studied variant, our study lacks statistical power to evaluate its association with outcome of CAP or IPD [7].

Once a PID is suspected in a patient, a molecular, confirmative diagnosis may help to make decisions about management, prognosis, and prophylactic measures, as well as to assist in genetic counseling. Cumulative evidence suggests that MASP-2, and even other components of the LP, are largely redundant in human defenses and that individuals with MASP-2 deficiency do not seem to be particularly prone to infectious or autoimmune diseases. Studies with very large groups of individuals would be required in order to firmly conclude whether MASP-2 deficiency could influence susceptibility to infection in individuals with other primary or secondary defects in components of the immune system.

Acknowledgments We are grateful to the patients and healthy individuals for their trust.

Author Contributions IG-L carried out experiments, analyzed data, critically discussed the results, and wrote the manuscript. EH-B carried out experiments and analyzed data. CM-A, SP-N, IR-F, OR, MLB, LB, AP, $\mathrm{LL}, \mathrm{JFr}, \mathrm{JFe}$, and IO provided clinical samples and data. NG-Q carried out experiments. FR and JS-V provided clinical samples and data and critically reviewed the manuscript. CR-G designed the work, critically discussed the results, and wrote the manuscript. All authors reviewed the final version of the manuscript.

Funding This work was partially supported by grants from the Instituto de Salud Carlos III, Ministry of Health (FIS PI13/01456 and PI16/00759 to CR-G, and PI13/01729 to CM-A, with the funding of European Regional Development Fund-European Social Fund -FEDER-FSE), from Grupo DISA (OA18/017) to CR-G, and from SEPAR (Spanish Society of Pulmonology and Thoracic Surgery-Fundación Española del Pulmón) to FR; EH-B was supported by a grant from Universidad de Las Palmas de Gran Canaria.
Data Availability All relevant data is contained within the manuscript. The raw datasets analyzed for this manuscript will be made available by the authors, without undue reservation, to any qualified researcher.

\section{Compliance with Ethical Standards}

Ethics Statement All procedures were carried out according to the Declaration of Helsinki, and approval was obtained from the Ethic Committee of the Hospital Universitario de Gran Canaria Doctor Negrin.

Informed Consent Informed consent was obtained from all individual participants included in the study

Conflict of Interest The authors declare that they have no conflicts of interest.

\section{References}

1. Ram S, Lewis LA, Rice PA. Infections of people with complement deficiencies and patients who have undergone splenectomy. Clin Microbiol Rev. 2010. https://doi.org/10.1128/CMR.00048-09.

2. Picard C, Bobby Gaspar H, Al-Herz W, Bousfiha A, Casanova JL, Chatila $\mathrm{T}$, et al. International Union of Immunological Societies: 2017 Primary Immunodeficiency Diseases Committee Report on Inborn Errors of Immunity. J Clin Immunol. 2018. https://doi.org/ 10.1007/s10875-017-0464-9.

3. Garred P, Genster N, Pilely K, Bayarri-Olmos R, Rosbjerg A, et al. A journey through the lectin pathway of complement-MBL and beyond. Immunol Rev. 2016. https://doi.org/10.1111/imr.12468.

4. Oroszlán G, Dani R, Szilágyi A, Závodszky P, Thiel S, Gál P, et al. Extensive basal level activation of complement mannose-binding lectin-associated serine protease-3: kinetic modeling of lectin pathway activation provides possible mechanism. Front Immunol. 2017. https://doi.org/10.3389/fimmu.2017.01821.

5. Dobó J, Kocsis A, Gál P. Be on Target: strategies of targeting alternative and lectin pathway components in complement-mediated diseases. Front Immunol. 2018. https://doi.org/10.3389/fimmu.2018.01851.

6. Stengaard-Pedersen K, Thiel S, Gadjeva M, Møller-Kristensen M, Sørensen R, Jensen LT, et al. Inherited deficiency of mannanbinding lectin-associated serine protease 2. N Engl J Med. 2003. https://doi.org/10.1056/NEJMoa022836.

7. Garcia-Laorden MI, Sole-Violan J, Rodriguez de Castro F, Aspa J, Briones ML, Garcia-Saavedra A, et al. Mannose-binding lectin and mannose-binding lectin-associated serine protease 2 in susceptibility, severity, and outcome of pneumonia in adults. J Allergy Clin Immunol. 2008. https://doi.org/10.1016/j.jaci.2008.05.037.

8. Notarangelo L, Casanova JL, Fischer A, Puck J, Rosen F, Seger R, et al. Primary immunodeficiency diseases: an update. J Allergy Clin Immunol. 2004. https://doi.org/10.1016/j.jaci.2004.06.044.

9. García-Laorden MI, García-Saavedra A, de Castro FR, Violán JS, Rajas O, Blanquer J, et al. Low clinical penetrance of mannosebinding lectin-associated serine protease 2 deficiency. J Allergy Clin Immunol. 2006. https://doi.org/10.1016/j.jaci.2006.08.004.

10. Hochberg MC. Updating the American College of Rheumatology revised criteria for the classification of systemic lupus erythematosus. Arthritis Rheum. 1997. https://doi.org/10.1002/art.1780400928.

11. Cedzynski M, Szemraj J, Swierzko AS, Bak-Romaniszyn L, Banasik $\mathrm{M}$, Zeman K, et al. Mannan-binding lectin insufficiency in children with recurrent infections of the respiratory system. Clin Exp Immunol. 2004. https://doi.org/10.1111/j.1365-2249.2004.02453.x.

12. Cedzynski M, Atkinson AP, St Swierzko A, MacDonald SL, Szala A, Zeman K, et al. L-ficolin (ficolin-2) insufficiency is associated with 
combined allergic and infectious respiratory disease in children. Mol Immunol. 2009. https://doi.org/10.1016/j.molimm.2009.08.028.

13. Sokolowska A, Szala A, St Swierzko A, Kozinska M, Niemiec T, Blachnio M, et al. Mannan-binding lectin-associated serine protease-2 (MASP-2) deficiency in two patients with pulmonary tuberculosis and one healthy control. Cell Mol Immunol. 2015;12:11921. https://doi.org/10.1038/cmi.2014.19.

14. Olesen HV, Jensenius JC, Steffensen R, Thiel S, Schiøtz PO. The mannan-binding lectin pathway and lung disease in cystic fibrosisdisfunction of mannanbinding lectin-associated serine protease 2 (MASP-2) may be a major modifier. Clin Immunol. 2006. https:// doi.org/10.1016/j.clim.2006.08.014.

15. Thiel S, Steffensen R, Christensen IJ, Ip WK, Lau YL, Reason IJ, et al. Deficiency of mannan-binding lectin associated serine protease-2 due to missense polymorphisms. Genes Immun. 2007. https://doi.org/10.1038/sj.gene.6364373.

16. Ytting H, Christensen IJ, Steffensen R, Alsner J, Thiel S, Jensenius JC, et al. Mannan-binding lectin (MBL) and MBL-associated serine protease 2 (MASP-2) genotypes in colorectal cancer. Scand J Immunol. 2011. https://doi.org/10.1111/j.1365-3083.2010.02480. $\mathrm{x}$.

17. Segat L, Fabris A, Padovan L, Milanese M, Pirulli D, Lupo F, et al. MBL2 and MASP2 gene polymorphisms in patients with hepatocellular carcinoma. J Viral Hepat. 2008. https://doi.org/10.1111/j.1365-2893. 2007.00965.x.

18. Siljan WW, Holter JC, Nymo SH, Husebye E, Ueland T, Skattum L, et al. Low levels of immunoglobulins and mannose-binding lectin are not associated with etiology, severity, or outcome in community-acquired pneumonia. Open Forum Infect Dis. 2018. https://doi.org/10.1093/ofid/ ofy002.

19. Stover C, Barrett S, Lynch NJ, Barker JN, Burden D, Trembath R, et al. Functional MASP2 single nucleotide polymorphism plays no role in psoriasis. Br J Dermatol. 2005. https://doi.org/10.1111/j. 1365-2133.2005.06547.x.

20. Olszowski T, Poziomkowska-Gęsicka I, Jensenius JC, Adler G. Lectin pathway of complement activation in a Polish woman with MASP-2 deficiency. Immunobiology. 2014. https://doi.org/10. 1016/j.imbio.2013.10.009.

21. Świerzko AS, Szala-Poździej A, Kilpatrick DC, Sobociński M, Chojnacka K, Sokołowska A, Michalski M, Mazerant K, Jensenius JC, Matsushita M, Krajewski WR, Szczapa J, BąkRomaniszyn L, Zeman K, Cedzyński M. Components of the lectin pathway of complement activation in paediatric patients of intensive care units. Immunobiology 2016;221(5):657-669.

22. Sørensen R, Thiel S, Jensenius JC. Mannan-binding-lectin-associated serine proteases, characteristics and disease associations. Springer Semin Immunopathol. 2005. https://doi.org/10.1007/s00281-0050006-z.

23. Casanova JL, Abel L. Human genetics of infectious diseases: Unique insights into immunological redundancy. Semin Immunol. 2018;36:1-12. https://doi.org/10.1016/j.smim.2017.12.008. Epub 2017 Dec 16.

24. Casanova JL, Conley ME, Seligman SJ, Abel L, Notarangelo LD. Guidelines for genetic studies in single patients: lessons from primary immunodeficiencies. J Exp Med. 2014. https://doi.org/10. 1084/jem.20140520.

25. Bousfiha A, Jeddane L, Picard C, Ailal F, Bobby Gaspar H, AlHerz W, et al. The 2017 IUIS phenotypic classification for primary immunodeficiencies. J Clin Immunol. 2018. https://doi.org/10. 1007/s10875-017-0465-8.

26. van Schaarenburg RA, Schejbel L, Truedsson L, Topaloglu R, AlMayouf SM, Riordan A, et al. Marked variability in clinical presentation and outcome of patients with $\mathrm{C} 1 \mathrm{q}$ immunodeficiency. J Autoimmun. 2015. https://doi.org/10.1016/j.jaut.2015.06.002.
27. Frank MM, Sullivan KE. Deficiencies of the complement system. In: Sullivan KE, Stiehm R, editors. Stiehm's immune deficiencies. Elsevier, Academic Press; 2014.pp. 731-763

28. Jönsson G, Oxelius VA, Truedsson L, Braconier JH, Sturfelt G, Sjöholm AG. Homozygosity for the IgG2 subclass allotype $\mathrm{G} 2 \mathrm{M}(\mathrm{n})$ protects against severe infection in hereditary $\mathrm{C} 2$ deficiency. J Immunol. 2006. https://doi.org/10.4049/jimmunol.177.1.722.

29. Verdu P, Barreiro LB, Patin E, Gessain A, Cassar O, Kidd JR, et al. Evolutionary insights into the high worldwide prevalence of MBL2 deficiency alleles. Hum Mol Genet. 2006. https:/doi.org/10.1093/ $\mathrm{hmg} / \mathrm{ddl1} 93$.

30. García-Laorden MI, Rodríguez de Castro F, Solé-Violán J, Payeras A, Briones ML, Borderías L, et al. The role of mannose-binding lectin in pneumococcal infection. Eur Respir J. 2013. https://doi. org/10.1183/09031936.00174111.

31. Luo J, Xu F, Lu GJ, Lin HC, Feng ZC. Low mannose-binding lectin (MBL) levels and MBL genetic polymorphisms associated with the risk of neonatal sepsis: An updated meta-analysis. Early Hum Dev. 2014. https://doi.org/10.1016/j.earlhumdev.2014.07.007.

32. Zhang AQ, Yue CL, Pan W, Gao JW, Zeng L, Gu W, et al. Mannose-binding lectin polymorphisms and the risk of sepsis: evidence from a meta-analysis. Epidemiol Infect. 2014. https://doi. org/10.1017/S0950268813003361.

33. Lundbo LF, Harboe ZB, Clausen LN, Hollegaard MV, Sørensen HT, Hougaard DM. Mannose-binding lectin gene, MBL2, polymorphisms are not associated with susceptibility to invasive pneumococcal disease in children. Clin Infect Dis. 2014. https://doi.org/ $10.1093 / \mathrm{cid} / \mathrm{ciu} 276$

34. Darton TC, Jack DL, Johnson M, Borrow R, Guiver M, Kaczmarski $\mathrm{EB}$, et al. MBL2 deficiency is associated with higher genomic bacterial loads during meningococcemia in young children. Clin Microbiol Infect. 2014. https://doi.org/10.1111/1469-0691.12745.

35. Mills TC, Chapman S, Hutton P, Gordon AC, Bion J, Chiche JD, et al. Variants in the mannose-binding lectin gene MBL2 do not associate with sepsis susceptibility or survival in a large European cohort. Clin Infect Dis. 2015. https://doi.org/10.1093/cid/civ378.

36. Lundbo LF, Sørensen HT, Clausen LN, Hollegaard MV, Hougaard DM, Konradsen HB, et al. Mannose-binding lectin gene, MBL2, polymorphisms do not increase susceptibility to invasive meningococcal disease in a population of Danish children. Open Forum Infect Dis. 2015. https://doi.org/10.1093/ofid/ofv127.

37. Bautista-Rodriguez C, Launes C, Jordan I, Andres M, Arias MT, Lozano F, et al. Mannose-binding lectin-deficient genotypes as a risk factor of pneumococcal meningitis in infants. PLoS One. 2017. https://doi.org/10.1371/journal.pone.0178377.

38. Al-Herz W, Bousfiha A, Casanova JL, Chapel H, Conley ME, Cunningham-Rundles C, et al. Primary immunodeficiency diseases: an update on the classification from the international union of immunological societies expert committee for primary immunodeficiency. Front Immunol. 2011. https://doi.org/10.3389/fimmu.2011.00054.

39. Ali YM, Lynch NJ, Haleem KS, Fujita T, Endo Y, Hansen S, et al. The lectin pathway of complement activation is a critical component of the innate immune response to pneumococcal infection. PLoS Pathog. 2012. https://doi.org/10.1371/journal.ppat.1002793.

40. Neth O, Jack DL, Dodds AW, Holzel H, Klein NJ, et al. Mannosebinding lectin binds to a range of clinically relevant microorganisms and promotes complement deposition. Infect Immun. 2000. https:// doi.org/10.1128/IAI.68.2.688-693.2000.

41. Howard M, Farrar CA, Sacks SH. Structural and functional diversity of collectins and ficolins and their relationship to disease. Semin Immunopathol. 2018;40:75-85. https://doi.org/10.1007/ s00281-017-0642-0.

42. Hwang I, Mori K, Ohtani K, Matsuda Y, Roy N, Kim Y, et al. Collectin kidney 1 plays an important role in innate immunity against Streptococcus pneumoniae infection. J Innate Immun. 2017. https://doi.org/10.1159/000453316. 
43. Rooryck C, Diaz-Font A, Osborn DP, Chabchoub E, HernandezHernandez V, Shamseldin H, et al. Mutations in lectin complement pathway genes COLEC11 and MASP1 cause 3MC syndrome. Nat Genet. 2011. https://doi.org/10.1038/ng.757.

44. Venkatraman Girija U, Furze CM, Gingras AR, Yoshizaki T, Ohtani K, Marshall JE, et al. Molecular basis of sugar recognition by collectin-K1 and the effects of mutations associated with 3MC syndrome. BMC Biol. 2015;13:1-14. https://doi.org/10.1186/s12915-015-0136-2.

45. Urquhart J, Roberts R, de Silva D, Shalev S, Chervinsky E, Nampoothiri S, et al. Exploring the genetic basis of 3MC syndrome: Findings in 12 further families. Am J Med Genet A. 2016. https://doi.org/10.1002/ajmg.a.37564.

46. Munye MM, Diaz-Font A, Ocaka L, Henriksen ML, Lees M, Brady $\mathrm{A}$, et al. COLEC10 is mutated in 3MC patients and regulates early craniofacial development. PLoS Genet. 2017. https://doi.org/10. 1371/journal.pgen.1006679.

47. Sirmaci A, Walsh T, Akay H, Spiliopoulos M, Sakalar YB, Hasanefendioğlu-Bayrak A, et al. MASP1 mutations in patients with facial, umbilical, coccygeal, and auditory findings of Carnevale, Malpuech, OSA, and Michels syndromes. Am J Hum Genet. 2010. https://doi.org/10.1016/j.ajhg.2010.09.018.

48. Atik T, Koparir A, Bademci G, Foster J 2nd, Altunoglu U, Mutlu GY, et al. Novel MASP1 mutations are associated with an expanded phenotype in 3MC1 syndrome. Orphanet J Rare Dis. 2015. https:// doi.org/10.1186/s13023-015-0345-3.

49. Degn SE, Jensen L, Hansen AG, Duman D, Tekin M, Jensenius JC, et al. Mannan-binding lectin-associated serine protease (MASP)- 1 is crucial for lectin pathway activation in human serum, whereas neither
MASP-1 nor MASP-3 is required for alternative pathway function. J Immunol. 2012. https://doi.org/10.4049/jimmunol.1201736.

50. Munthe-Fog L, Hummelshøj T, Honoré C, Madsen HO, Permin H, Garred P. Immunodeficiency associated with FCN3 mutation and ficolin-3 deficiency. N Engl J Med. 2009. https://oi.org/10.1056/ NEJMoa0900381.

51. Michalski M, Świerzko AS, Pagowska-Klimek I, Niemir ZI, Mazerant K, et al. Primary ficolin-3 deficiency-is it associated with increased susceptibility to infections? Immunobiology. 2015. https://doi.org/10.1016/j.imbio.2015.01.003.

52. Troldborg A, Steffensen R, Trendelenburg M, Hauser T, Winther KG, Hansen AG, et al. Ficolin-3 deficiency is associated with disease and an increased risk of systemic lupus erythematosus. J Clin Immunol. 2019;39:421-9. https://doi.org/10.1007/s10875-019-00627-2.

53. Banda NK, Acharya S, Scheinman RI, Mehta G, Takahashi M, Endo Y, et al. Deconstructing the lectin pathway in the pathogenesis of experimental inflammatory arthritis: essential role of the lectin ficolin B and mannose-binding protein-associated serine protease 2 . J Immunol. 2017. https://doi.org/10.4049/jimmunol.1700119.

54. Kasanmoentalib ES, Valls Seron M, Ferwerda B, Tanck MW, Zwinderman AH, Baas F, et al. Mannose-binding lectin-associated serine protease 2 (MASP-2) contributes to poor disease outcome in humans and mice with pneumococcal meningitis. J Neuroinflammation. 2017;14:110. https://doi.org/10.1186/s12974-016-0770-9.

Publisher's Note Springer Nature remains neutral with regard to jurisdictional claims in published maps and institutional affiliations.

\section{Affiliations}

\section{Isabel García-Laorden ${ }^{1,2}$ • Elisa Hernández-Brito ${ }^{3,4}$. Carmen Muñoz-Almagro ${ }^{5,6,7}$ - Svetlana Pavlovic-Nesic ${ }^{8}$. Iñigo Rúa-Figueroa ${ }^{9} \cdot$ M. Luisa Briones ${ }^{10} \cdot$ Olga Rajas $^{11} \cdot$ Luis Borderías $^{12} \cdot$ Antoni Payeras $^{13} \cdot$ Leonardo Lorente $^{14}$. Jordi Freixinet ${ }^{15}$. Jose Ferreres ${ }^{16}$. Ignacio Obando ${ }^{17}$ • Nereida González-Quevedo ${ }^{3}$. Felipe Rodríguez de Castro ${ }^{4,18}$. Jordi Solé-Violán ${ }^{1,19} \cdot$ Carlos Rodríguez-Gallego ${ }^{3,20}$}

1 CIBER de Enfermedades Respiratorias (CIBERES), Instituto de Salud Carlos III, Madrid, Spain

2 Research Unit, Hospital Universitario de Gran Canaria Dr. Negrín, Las Palmas de Gran Canaria, Spain

3 Department of Immunology, Hospital Universitario de Gran Canaria Dr. Negrín, Las Palmas de Gran Canaria, Spain

4 Department of Medical and Surgical Sciences, School of Medicine, Universidad de Las Palmas de Gran Canaria, Las Palmas de Gran Canaria, Spain

5 Paediatric Infectious Diseases Research Group, Institut de Recerca Sant Joan de Déu, Esplugues de Llobregat, Spain

6 CIBER en Epidemiología y Salud Pública (CIBERESP), Instituto de Salud Carlos III, Madrid, Spain

7 School of Medicine, Universitat Internacional de Catalunya, Barcelona, Spain

8 Department of Pediatrics, Complejo Hospitalario Universitario Insular Materno Infantil, Las Palmas de Gran Canaria, Spain

9 Rheumatology Service, Hospital Universitario de Gran Canaria Dr. Negrín, Las Palmas de Gran Canaria, Spain
10 Department of Respiratory Diseases, Hospital Clínico y Universitario de Valencia, Valencia, Spain

11 Department of Respiratory Diseases, Hospital Universitario de la Princesa, Madrid, Spain

12 Department of Respiratory Diseases, Hospital San Jorge, Huesca, Spain

13 Department of Internal Medicine, Hospital Son Llatzer, Palma de Mallorca, Spain

14 Intensive Care Unit, Hospital Universitario de Canarias, La Laguna, Spain

15 Department of Thoracic Surgery, Hospital Universitario de Gran Canaria Dr. Negrín, Las Palmas de Gran Canaria, Spain

16 Intensive Care Unit, Hospital Clínico y Universitario de Valencia, Valencia, Spain

17 Department of Pediatrics, Hospital Virgen del Rocío, Sevilla, Spain

18 Department of Respiratory Diseases, Hospital Universitario de Gran Canaria Dr. Negrín, Las Palmas de Gran Canaria, Spain 